\title{
Improving viable low cost generic medication prescription rate in primary care pediatric practice
}

\author{
Sathyanarayan Sudhanthar, Jane Turner, Kripa Thakur, Yakov Sigal \\ Dept. of Pediatrics, College of Human Medicine, Michigan State University, USA
}

\begin{abstract}
The pediatric clinics of the Michigan State University College of Human Medicine (MSU-CHM) consist of academic pediatricians serving two clinics with a patient population of 5200 . The internal quality measures published by the MSU health team had consistently indicated our generic medications prescription rate to be very low, with an average of about $21 \%$ for the years 2009,2010 , and 2011 . There was an earnest need to increase our generic medication prescription rates, which is considered an indicator of good practice. The stakeholders identified were physicians, nurses, care managers, and the health information technology (HIT) team.
\end{abstract}

The purpose of the project was to increase the prescribing rate of viable low cost and approved generic medications for the patients of the MSU-CHM general pediatric clinics.

The stakeholders regularly met every few months to work on increasing the generic medication prescription rates based on the PDSA cycle. Help was sought from HIT to identify and acquire the top 10 brand names the group was prescribing along with individual provider data. The team reviewed the brand names most prescribed, and made a recommendation that the best group to target were the stimulant medications, allergy and asthma medications, and other psychotropics. The HIT team was then requested to add the available generics for all stimulant medications, asthma medications, and others in the electronic medical record (EMR). They were also clearly marked for ease of use, for example: amphetamine-dextroamphetamine extended release "generic for Adderall XR." It was decided that providers would prescribe all stimulants as a generic, unless not available, and nurses would change each brand name of stimulants to a generic every time they refilled a medicine, based on a protocol outlining the appropriate generic medications corresponding to the respective brand names. The physicians and nurses were also urged to discuss with the patients the substitution process and answer any questions from parents. Monthly reports were obtained from the HIT about our progress.

After 12 months of implementing this project, the overall generic prescription rate increased from $20 \%$ at the end of first quarter 2012 to $53 \%$ at the end of 12 months, and $65.5 \%$ at the end of two years. This was well above the MSU health team (about six large group practices) primary care average of $34.6 \%$. All brand name medication prescription rates were also decreased. This is a positive outcome for this project in a relatively short period of time, and a further plan will be to repeat the cycle and continue to improve on the generic prescription rate, thereby saving valuable dollars spent on health care.

\section{Problem}

Internal review of the Michigan State University (MSU) health team revealed that the College of Human Medicine (CHM) pediatric clinics lagged behind severely in prescribing generic medications, compared to their peers of other primary care large group practices, including family medicine, family practice, internal medicine, and specialty practices such as women's health. The overall average generic medication prescribing rate was $34 \%$ to $35 \%$ for the health team, whereas the rate was $21 \%$ for the $\mathrm{CHM}$ pediatric clinics. Providers were prescribing high cost brand name medications in spite of the availability of low cost, viable approved generic medications. Apart from the impact on the patient family for the copay of these medications, there were multiple denials from insurance companies to cover the high cost brand names. Hence the staff had to convert these to low cost brand names or get prior authorization from the insurance companies, which was creating a huge impact on workflow and the efficient use of staff time. Most providers tend to remember brand names better than generic names due to advertisements, flyers, and also because of the easy searchable feature in the electronic medical record (EMR). Hence, providers and staff at $\mathrm{CHM}$ pediatric clinics wanted to work together to improve the service and save valuable dollars spent on brand name medications.

\section{Background}

The cost of prescription drugs as a part of total health care spending has been growing significantly across the world.[1] The per capita national health expenditure in the United States in 2013 was $\$ 9250$, and the total national health expenditure was $\$ 2.9$ trillion.[2] Prescription costs in the United States account for $9.3 \%$ of total expenditure, which is about $\$ 290 \mathrm{bn}$ to $\$ 300 \mathrm{bn}$. An average American consumer is likely to use about 12.2 prescriptions per year. Generic medications cost significantly less in copay $(50 \%$ less) than brand name medications, and the savings may be more if medications are bought in retail.[3] This is especially true in the global market, as one study from India estimates the cost savings 
for the average consumer could be up to $90 \%$ compared to brand name medications.[4] Another study estimates by switching just four medications to their generic equivalents China could save $\$ 370 \mathrm{~m}$, and the average cost savings by switching to generics would be $10 \%$ to $89 \%$ on the most common medications prescribed.[5] In the United States the cost for prescriptions is on the rise, and has risen significantly in the past decade.[6] In the United Kingdom it is a standard practice to prescribe generics, as $83 \%$ of the medications are dispensed in generic form.[7] There have been many health care policies carried out in countries such as Finland and Sweden, where generic substitutions are mandatory by the pharmacy and can be denied only by physician or patient preference. The cost saving in these countries is very significant, and estimated to be about $60 \%$ to $65 \%$ over brand name medications. $[10,11]$ In addition, Australia has introduced several health policies including incentives and a price reduction system for medications to be placed on the formulary, which is estimated to save their health care about $\$ 6.9$ billion over the next 10 years.[10]

\section{Baseline measurement}

To obtain a specific baseline measurement, we obtained data on the top 10 brand name medications prescribed across all providers in both clinics. The top six most prescribed brand name medications were controlled substances used for attention deficit hyperactivity disorder (ADHD). Medications which do not have generics, for example asthma medications such as Proair and Flovent, were not included in the baseline measurement. We also obtained the overall rate of generic prescription across all visits, which was $28 \%(n=615)$ for the last quarter of 2012. The rates from prior years obtained from the health team data showed generic prescribing rates to be an average of $20 \%$ for 2010 and $21.5 \%$ for 2011 . Our providers were already doing a competent job in prescribing antibiotics in their generic form compared to the controlled substance medications and psychotropics.

See supplementary file: ds6546.docx - "Table 1: brand name prescriptions before and after intervention; Table 2: yearly generic prescriptions by percentage, 2010-14"

\section{Design}

The first step of the project was to identify the responsible stakeholders. Physician providers, nurses who refilled medications, care managers, and representatives from the health information technology (HIT) team were all identified as stakeholders. The team met every two to three months and started to work on strategies to improve the generic prescription rate. It was decided to collect baseline data about the top 10 brand name medications that were prescribed by the providers. Based on that data a plan was formulated to target medications for ADHD, asthma, and allergies. HIT was requested to add the generic names for all the stimulants in a way that was easily searchable and recognizable. The names had a tag after them that indicated they were generic to a specific brand name. A clinic flow protocol was developed where the providers consciously started prescribing generic medications for the brand names when available, and since the nursing staff handled most of the refills for patients with ADHD and other conditions they were requested to change all brand names into generic medications. Patients' families were also informed of the change and assured that these changes would be monitored, and that all the generic medications were approved, low cost, and had comparable therapeutic efficacy. Quarterly reports were obtained from the MSU HIT team to monitor the progress.

\section{Strategy}

PDSA cycle 1: The stakeholders met every other week for one to two hours and started to work on methods to increase the generic prescription rates based on PDSA cycle. It was decided that nursing staff and physicians would prescribe new medications as generic equivalents, and nursing staff would try to switch the brand name medications to generic equivalents when they refilled medications for the patients.

PDSA cycle 2: The effect of first cycle was very minimal, and hence the team reviewed the brand names most prescribed across all providers and made a recommendation that the best groups to target would be the stimulant medications, asthma medications, and allergy medications. The HIT team was then requested to add the available generics for all stimulant medications, asthma medications, allergy medications, and antibiotics in the EMR.

PDSA cycle 3:The staff and providers experienced difficulty in finding the appropriate generic names in the EMR, and hence HIT was requested to rename the generics and clearly mark for ease of use, for example amphetamine-dextroamphetamine extended release "generic for Adderall XR."

PDSA cycle 4: A clinic flow protocol was developed wherein nurses changed each brand name stimulant to a generic every time they refilled a medicine. The protocol stated the most appropriate medications for certain brand names. The physicians and nurses were also urged to discuss with the patients the substitution process and answer any questions from parents. Quarterly reports were obtained from the HIT about their progress.

\section{Results}

After implementing the project over eight quarters, the overall rate of prescribing generic medications for all clinical visits improved from an average of $21 \%$ from the prior years to $53 \%$ at the end of 12 months and $65.5 \%$ at the end of two years. The improvement was seen across all providers at the MSU-CHM pediatric clinics and in both of our clinical sites. Prescribing of the top eight brand name medications decreased significantly. The estimated cost implications calculated just for our top three brand name drugs switched to generics is about $\$ 53800$ for four quarters and about $\$ 100000$ across the age of the project.

See supplementary file: ds6583.pptx - "Rate of generic prescriptions and cost impact"

\section{Lessons and limitations}


There was some resentment from providers and nursing staff as they had to remember the generic names instead of brand names, which was a difficult task for most. As the clinic flow protocol was developed which stated the appropriate generic medication to be substituted for a particular brand name, there seemed to be more commitment from all. This project would not have been possible if not for the continued dedication among all stakeholders, especially the HIT. Reliable communication and follow up was imperative for such a project to succeed. There were concerns among patients when they suddenly saw their brand names were switched to generic equivalents, and the providers and nursing staff had to reassure the patients. We also ran into a unique problem of one insurance payer covering the brand name of amphetaminedextroamphetamine and not the generic equivalent. Since there are always questions about the comparison of therapeutic efficacy between brand and generic equivalents, active measures were put forward to follow up with patients if there were any concerns.

\section{Conclusion}

This project had a positive impact on the prescription rate of viable low cost approved generic equivalents instead of brand name medications. The plan will be to continue to improve the generic prescribing across all the clinics in the MSU health team based on this model. The rate of generic prescription improved significantly across all the providers in both clinics. This is also a good example of how information technology (the EMR) could be used to the advantage of the health care system and drive the cost of health care delivery. The cost impact of such projects is significant in health care and is a suitable example of system based practice. Ultimately, generic medication utilization and cost savings will be dependent on various factors including availability of therapeutically equivalent generic medications, communication and commitment from pharmacies and physicians, physician commitment to keep health care costs low, patient education on the utilization of generic medications and the individual and global cost savings to health care, rigorous monitoring of the quality of generic medications, and financial incentives to the health care providers and pharmacies to switch to generics.[11,12]

\section{References}

1. Daemmrich A, Mohanty A. Healthcare reform in the United States and China: Pharmaceutical market implications. J Pharm Policy Pract 2014 14;7(1):9.

2. National Center for Health Statistics. Health, United States, 2014: With Special Feature on Adults Aged 55-64. Hyattsville, MD: 2015. p.304-5. http://www.cdc.gov/nchs/data/hus/hus14.pdf\#103

3. IMS institute for health care informatics. Medicine Use and Shifting Costs of Healthcare: A Review of the Use of Medicines in the U.S. in 2013. April 2014. http://www.imshealth.com/en/thought-leadership/imsinstitute/reports/use-of-medicines-in-the-us-2013

4. Mathew P. Generic drugs: Review and experiences from South India. J Family Med Prim Care 2015;4(3):319-23.

5. Cameron A, Mantel-Teeuwisse AK, Leufkens HG, Laing
RO. Switching from originator brand medicines to generic equivalents in selected developing countries: how much could be saved? Value Health 2012 15(5):664-73.

6. Hoffman JM, Li E, Doloresco F, et al. Projecting future drug expenditures--2012. Am J Health Syst Pharm 2012 69(5):405-21.

7. Duerden MG, Hughes DA. Generic and therapeutic substitutions in the UK: Are they a good thing? $\mathrm{Br} J$ Clin Pharmacol 2010 70(3):335-41.

8. Martikainen J., Rajaniemi S. Drug reimbursement systems in EU member states, Iceland and Norway. Helsinki: The Social Insurance Institution, Finland, Social security and health reports 2002;54:36.

9. Andersson K, Sonesson C, Petzold M, Carlsten A, Lönnroth $\mathrm{K}$. What are the obstacles to generic substitution? An assessment of the behaviour of prescribers, patients and pharmacies during the first year of generic substitution in Sweden. Pharmacoepidemiol Drug Saf 2005;14(5):341-8.

10. The Centre for Strategic Economic Studies (CSES). The Impact of PBS Reforms on PBS Expenditure and Savings Victoria University: Australia, 2009. http://main.medaus.com.au/files/2010/02/The-impact-ofPBS-reforms-on-PBS-expenditure-and-savings.pdf

11. Hassali MA, Alrasheedy AA, McLachlan A, et al. The experiences of implementing generic medicine policy in eight countries: A review and recommendations for a successful promotion of generic medicine use. Saudi Pharm J 2014;22(6):491-503.

12. Sheppard A. Generic medicines: Essential contributors to the long-term health of society. Ims Health 2011. http://www.egagenerics.com/images/Website/IMS Generic Medicines_Essential_contributors.pdf

\section{Declaration of interests}

None to declare

\section{Acknowledgements}

Primary care MSU-CHM pediatric team including Jonathan Gold M.D; Olga Napolova M.D; Jessica Gengler D.O; Michael Stiffler M.D; nursing staff, and the MSU health team.

\section{Ethical approval}

According to the Institutional Review Board at the Michigan State University "This project is deemed exempt as federal regulations for protection of human subjects will not apply to the project as this project is aimed to decrease the cost to the patient and health care in general." MSU IRB determined that the project did not need their approval 